\title{
Grant No.DE-FG02-01ER15203: Physics of Low-Dimensional Bose-Einstein Condensates
}

A final technical report submitted to the U.S. Department of Energy, Office of Basic Energy Sciences, Chemical Sciences, Geosciences and Biosciences Division

Eugene B. Kolomeisky

\author{
Department of Physics \\ University of Virginia \\ 382 McCormick Road \\ P.O. Box 400714
}

Charlottesville, Virginia 22904-4714 
This document is a final technical report submitted to the Chemical Sciences, Geosciences and Biosciences Division, Office of Basic Energy Sciences, Office of Science, U. S. Department of Energy summarizing theoretical work conduncted under current Nanoscale Science, Engineering and Technology Initiative Research Grant No.DE-FG02-01ER15203 entitled Physics of Low-Dimensional Bose-Einstein Condensates.

As of today the work supported by the current Department of Energy Research Grant resulted in six publications; there are also two manuscripts submitted for publication and two more papers will be submitted for publication before the current funding cycle expires. Total of four graduate students (Xiaoya Qi, Ryan M. Kalas, Michael Timmins, and Kangkang Li) have been involved with various aspects of the research as a part of their Ph.D training. Mr. Qi, Mr. Kalas and Mr. Timmins defended their Ph.D theses in May 2003, August 2004, and July 2005, respectively. Mr. Kalas is currently a Postdoctoral Research Associate at the Washington State University. Ph.D plans of Ms. Li are not yet clear as she has been in the PI research group for only a year. Additionally a partial support was provided for Dr. Xiao Yang who is a Postdoctoral Research Associate shared between the PI and Professor Fendley of the UVA Physics Department.

- Statement of Unexpended Funds: it is expected that no funds will be left by the end of the current funding cycle.

Our results have been delivered at the Rutgers Statistical Mechanics Meetings (X. Qi in Fall 2001 and R. M. Kalas in Spring 2002), the 2002 American Physical Society Meeting (R. M. Konik), and International Conference on Cosmology in Laboratory (E. B. Kolomeisky in Summer 2003). The PI has also been invited to give talks at the Institute of Theoretical Physics, ETH in Zürich, Switzerland (2003), University of Kentucky (2003), and to participate in a workshop Quantum Gases which took place April 26 - May 15, 2004 in the Kavli Institute of Theoretical Physics, University of California at Santa Barbara.

A more specific account of published work supported by the current Department of Energy Research Grant is given below in chronological order:

- E. B. Kolomeisky, R. M. Konik, and X. Qi, Quantum fluctuations of charge and phase transitions of a large Coulomb-blockaded quantum dot, Phys. Rev. B 66, 075318 (2002). Quantum dots are nanometer-scale structures hosting a few to a few thousand electrons and coming in a variety of forms. A useful mental picture of a dot is that of a container of electrons connected to a reservoir through a tunneling junction and placed nearby a gate electrode. The role of the gate electrode is to create favorable electrostatic conditions for the dot to host a desirable number of electrons. This number is controlled experimentally and is not necessarily integer. On the other hand, the electrons are discrete, and can only enter the dot one at a time. As a result, the electrons enter the dot only at special values of the gate voltage, and most of the time the dot population does not respond to changes in the gate voltage. This phenomenon predicted in the mid 80 's and first observed in the early 90's is called Coulomb blockade. One of its hallmarks is the dependence of the population of the dot on the gate voltage consisting of plateaus corresponding to an integer number of electrons on the dot - the celebrated Coulomb staircase.

This classical picture is modified qualitatively even at zero temperature because in reality the dot is never completely isolated from the reservoir. Earlier studies concluded that even infinitesimally weak coupling between the dot and reservoir will smear the Coulomb staircase. In our work the whole problem is reexamined for the case when the system is placed in a strong magnetic field (the electron spin is effectively zero), and there is an electron-electron interaction.

We found that the old conclusion that the Coulomb staircase will be always smeared is just one of the possible answers, and the classical step-like dependence of the average number of electrons on the dot $n$ as a function of the (dimensionless) gate voltage $n_{x}$ is preserved under certain conditions, the presence of quantum fluctuations notwithstanding. We pointed out that in its low-energy limit the problem is dual to that of a single-junction SQUID with Caldeira-Leggett dissipative coupling, and analogous to the classical problem of multilayer adsorption, and so is related to the classical statistical-mechanical problem of a one-dimensional Ising model with exchange interactions decaying as the inverse-square of distance. This Ising universality class further subdivides into what we termed (i) the Kondo/Ising class and (ii) the tricritical class.

(i) For systems of the Kondo/Ising class, the $n\left(n_{x}\right)$ dependence is always continuous for attractive electron-electron interactions, while if the electrons repel each other the $n\left(n_{x}\right)$ dependence is continuous for sufficiently open dots, while taking the form of a modified staircase for dots sufficiently isolated from the reservoir. At the phase transition between the two regimes the magnitude of the dot population jump is 
(a)

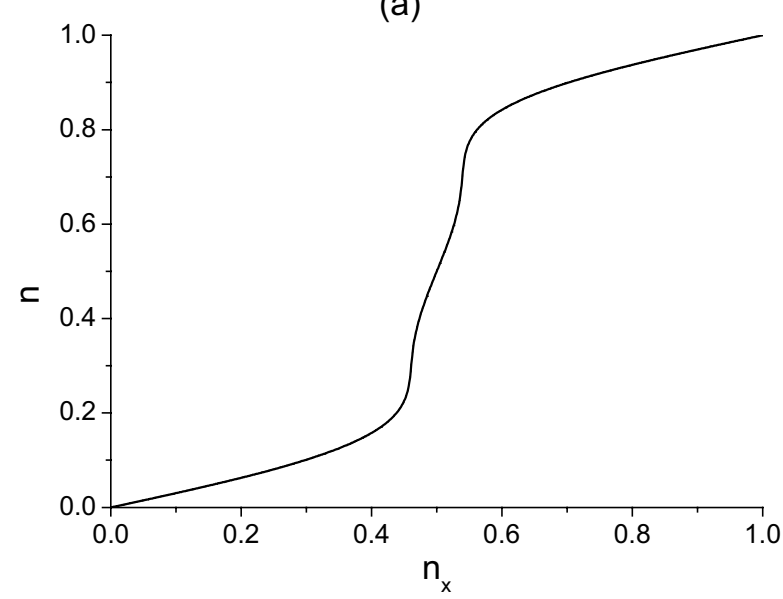

(b)

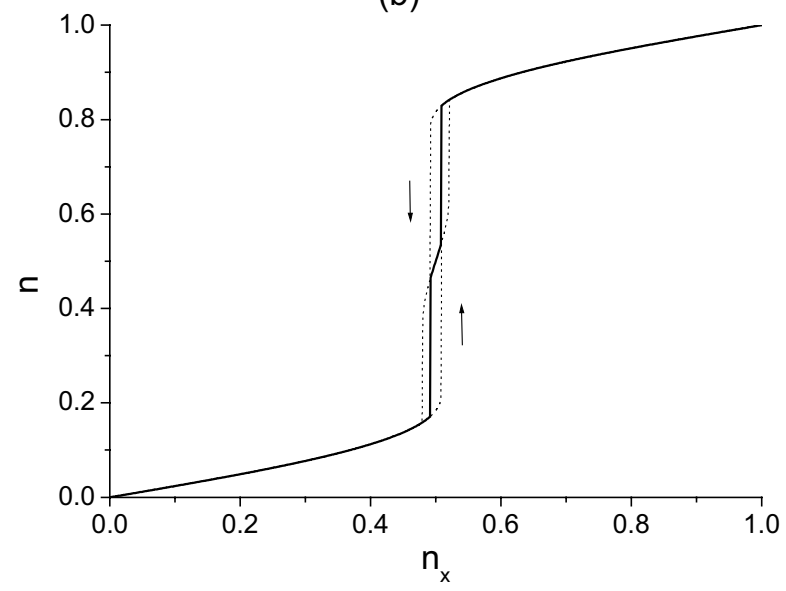

(c)

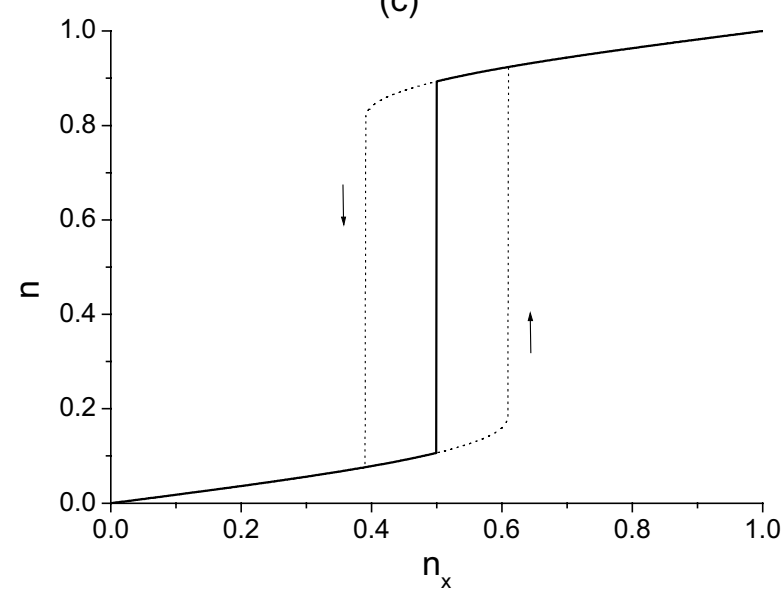

Figure 1: Average number (modulo one) of electrons on the dot as a function of the gate voltage $n_{x}$ as explained in the text. Hysteresis loops corresponding to non-adiabatic variation of the gate voltage are shown by the dotted lines and the direction of change of $n_{x}$ is indicated by the arrows. 


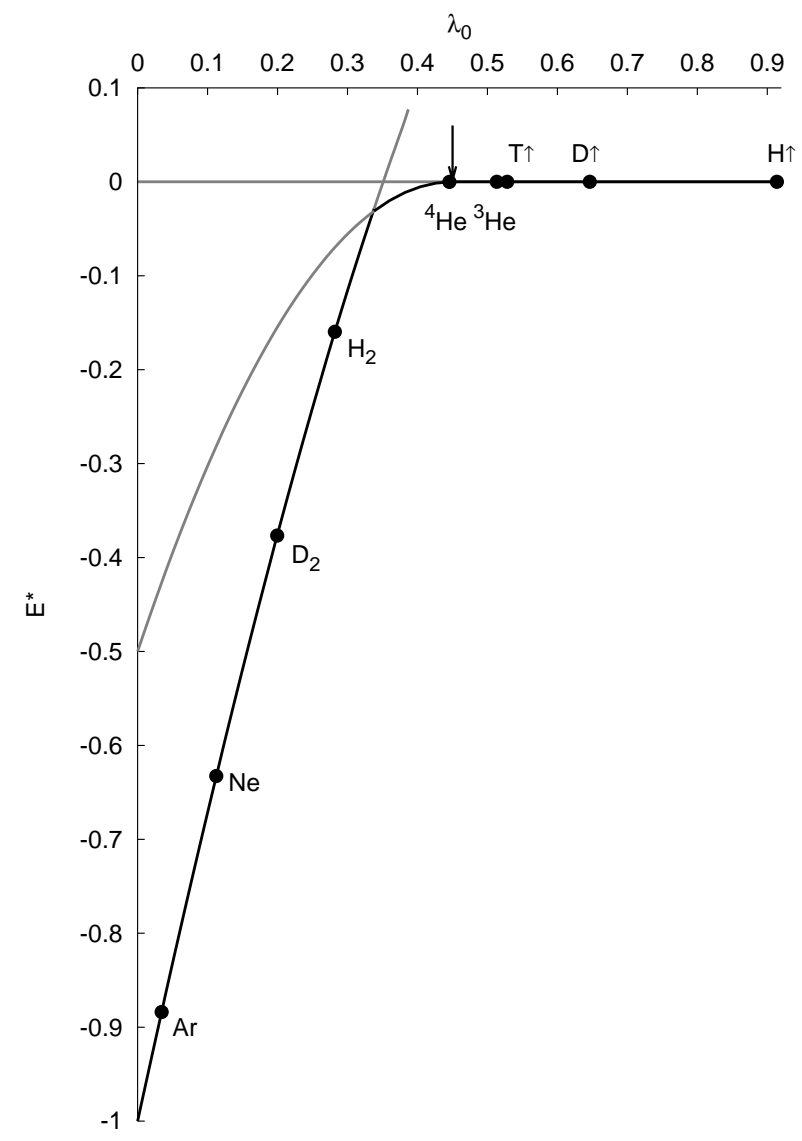

Figure 2: The dependencies of the reduced energy per particle on De Boer's number $\lambda_{0}$ for various phases. The bold parts of the curves correspond to ground states of the system while the gray scale segments indicate metastable states. The loci of a series of substances are shown by solid dots, and the arrow pointing down is the dimer dissociation threshold.

only determined by the properties of the reservoir and thus is universal.

(ii) For systems in the tricritical class we found in addition an intermediate regime where the dot population jumps from near integer value to a region of stable population centered about a half-integer value. In particular, this tricritical behavior (together with the two dependencies already seen in the Kondo/Ising class) is realized for non-interacting electrons.

As a sample result in Fig.1 we show the dependence of the average population of the dot on the gate voltage for strong (a), intermediate (b), and weak coupling to the reservoir (c). The electrons are taken to be moderately repulsive and the details can be found in the original paper.

Our paper has been selected for the August 15, 2002 issue of the Virtual Journal of Applications of Superconductivity, http://www.vjsuper.org and for the August 26, 2002 issue of the Virtual Journal of Nanoscale Science and Technology, http://www.vjnano.org.

- E. B. Kolomeisky, X. Qi, and M. Timmins, Ground-state properties of one-dimensional matter and quantum dissociation of a Luttinger liquid, Phys. Rev. B 67, 165407 (2003).

Ever since the experimental discovery of carbon nanotubes in 1991 [1] studying the properties of onedimensional systems became especially important. In addition to their unique transport, mechanical and chemical properties [2], bundles of carbon nanotubes can play a role of one-dimensional hosts for foreign atoms that can find themselves bound in the interstitial channels or inside the tubes [3]. One of the interesting potential applications of these systems includes storage devices for molecular hydrogen in fuel cells [4].

Motivated by these experimental possibilities, we analyzed ground-state properties of strictly one-dimensional 
molecular matter comprised of identical particles of mass $m$. Such a class of systems can be described by an additive two-body potential whose functional form is common to all substances which only differ in the energy $\epsilon$ and range $l$ scales of the potential. With this choice De Boer's quantum theorem of corresponding states holds [5], and the ground-state properties expressed in appropriate reduced form are only determined by the dimensionless parameter $\lambda_{0}^{2} \sim \hbar^{2} / m l^{2} \epsilon$ measuring the strength of zero-point motion in the system. The presence of a minimum in the two-body interaction potential leads to a many-body bound state which is a Luttinger liquid stable for a not very large $\lambda_{0}$. As $\lambda_{0}$ increases, the asymmetry of the two-body potential causes quantum expansion, softening, and eventual evaporation of the Luttinger liquid into a gas phase. Selecting the pair interaction potential in the Morse form we analytically computed the properties of the Luttinger liquid and its range of existence. We found that as $\lambda_{0}$ increases, the system first undergoes a discontinuous evaporation transition into a diatomic gas followed by a continuous dissociation transition into a monoatomic gas.

Fitting two-body potentials of molecular substances into the Morse form provided us with the depth of the potential well $\epsilon$ and the interaction range $l$. Supplemented by the masses of the underlying particles, this information is an input of our theory which then allows to determine De Boer's quantum parameter $\lambda_{0}$, the ground state and virtually any property. In particular we found that spin-polarized isotopes of hydrogen and ${ }^{3} \mathrm{He}$ are monoatomic gases, ${ }^{4} \mathrm{He}$ is a diatomic gas, while molecular hydrogen and heavier substances are Luttinger liquids. These results are illustrated in Fig. 2 where we show the reduced ground-state energy per particle as a function of De Boer's number $\lambda_{0}$ for various states of matter in question.

We also investigated the effect of finite pressure on the properties of the liquid and monoatomic gas phases. In particular we estimated a pressure at which molecular hydrogen undergoes an inverse Peierls transition into a metallic state which is a one-dimensional analog of the transition predicted by Wigner and Huntington in 1935.

The approach based on the quantum theorem of corresponding states was originally applied to predict the properties of ${ }^{3} \mathrm{He}[5]$ before it had become experimentally available. Later Anderson and Palmer [6] and Clark and Chao [7] used the same idea to estimate the properties of zero-temperature nuclear and neutronstar matter from those of laboratory substances. What distinguishes our work is that in one dimension we managed to proceed analytically and predict the properties of one-dimensional molecular matter from first principles.

Our study had a by-product which in our opinion warrants further investigation: for the first time we derived a condition resembling Lindemann's empirical criterion of melting [8] thus shedding new light on mechanisms of melting.

- J. P. Straley, E. B. Kolomeisky, and S. C. Milne, The Bose molecule in one dimension, Journal of Statistical Physics, 116, 1579 (2004). In this paper we considered a collection of $N$ identical bosons in one dimension, with an attractive delta-function interaction. There is a bound state for all $N$, having a form of a well-localized "molecule". This result is interesting, since it is a unique example of an exactly solved localized many-body problem.

This system has recently found an experimental realization in a ${ }^{7} \mathrm{Li}$ vapor [9] confined to an atomic trap which is so tight in two directions, that the system can be regarded as one-dimensional with respect to the third direction. In this system the $L i$ atoms are bosons with an interaction which can be attractive, resulting in a collapse. In this context, the boson molecule is called a "bright soliton".

For this problem we computed exactly the Green function, momentum distribution, two-particle correlation function, and structure factor. We also gave an argument showing that this boson "molecule" has no excited states other than dissociation into separate pieces.

- E. B. Kolomeisky, J. P. Straley, and R. M. Kalas, Ground-state properties of artificial bosonic atoms, Bose interaction blockade and the single-atom pipette, Phys. Rev. A, 68, 063401 (2004). We analyzed the ground-state properties of an artificial atom made out of repulsive bosons attracted to a center for the case that all the interactions are short-ranged. Such bosonic atoms could be created by optically trapping ultracold particles of alkali vapors; we presented the theory describing how their properties depend on the experimentally adjustable strength of "nuclear" attraction and interparticle repulsion. The binding ability of the short-range potential increases with space dimensionality - only a limited number of particles can be bound in one dimension, while in two and three dimensions the number of bound bosons can be chosen at will. Particularly in three dimensions we found an unusual effect of enhanced resonant binding: 

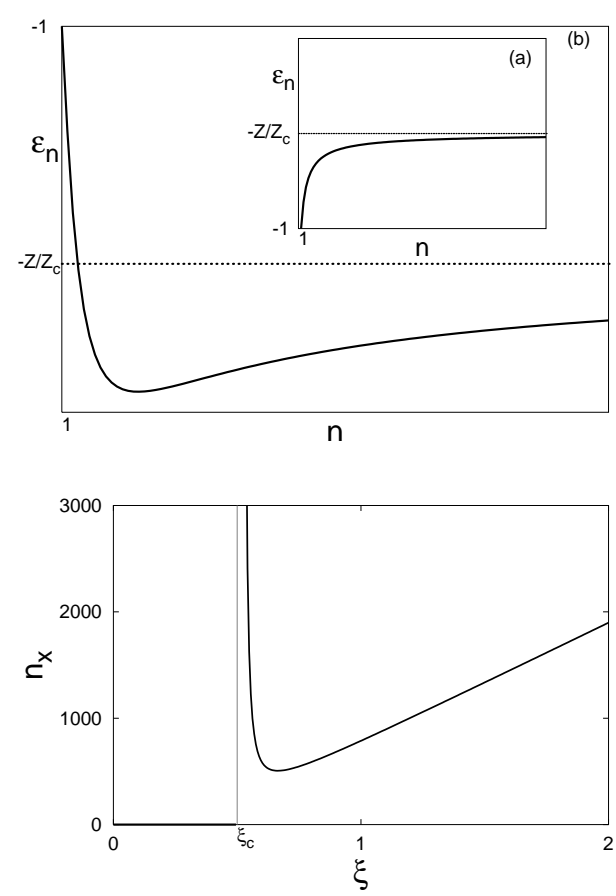

Figure 3: Ground-state energy of a three-dimensional Bose-atom as a function of number of bound particles: (a) strongly repulsive particles, (b) weakly repulsive particles.

Figure 4: Typical dependence of the equilibrium number of bound particles in a three-dimensional Bose-atom as a function of dimensionless depth of the nuclear potential $\xi$ for weak interparticle repulsion.

for not very strong interparticle repulsion the equilibrium number of bosons bound to a nuclear potential having a sufficiently shallow single-particle state increases without bound as the nuclear potential becomes less attractive. Our result is summarized in Figures 3 and 4.

As a consequence of the competing nuclear attraction enhanced by the Bose statistics and interparticle repulsions, the dependence of the ground-state energy of the atom on the number of particles has a minimum whose position is experimentally tunable. This implies a staircase dependence of the equilibrium number of bound bosons on external parameters which may be used to create a single-atom pipette - an arrangement which allows the transport of atoms into and out of a reservoir, one at a time.

- T.J. Newman, E.B. Kolomeisky, and J. Antonovics, Population dynamics with global regulation: The conserved Fisher equation, Phys. Rev. Lett. 93, 228103 (2004). We introduced a conserved version of the Fisher equation. Within a population biology context, this model describes spatially extended populations in which the total number of individuals is fixed due to either biotic or environmental factors. We found a rich spectrum of dynamical phases including a pseudo-traveling wave, and in the presence of the Allee effect, a phase transition from a locally constrained high-density state to a low-density fragmented state.

This work is not immediately related to the physics of low-dimensional quantum liquids except for the fact that virtually the same mathematics also describes an Abrikosov flux line in a deformable medium.

- E.B. Kolomeisky and M. Timmins, The Zel'dovich effect and evolution of atomic Rydberg spectra along the Periodic Table, Phys. Rev. A, to be published (2004), http://arxiv.org/abs/physics/0504154. In 1959 Ya. B. Zel'dovich predicted that the bound-state spectrum of the non-relativistic Coulomb problem distorted at small distances by a short-range potential undergoes a peculiar reconstruction whenever this potential alone supports a low-energy scattering resonance. However documented experimental evidence of this effect has been lacking. Previous theoretical studies of this phenomenon were confined to the regime where the range of the short-ranged potential is much smaller than Bohr's radius of the Coulomb field. We go beyond this limitation by restricting ourselves to highly-excited $s$ states. This allows us to demonstrate that along the Periodic Table of elements the Zel'dovich effect manifests itself as systematic periodic variation of the Rydberg spectra with a period proportional to the cubic root of the atomic number $Z$. This dependence, which is supported by analysis of experimental and numerical data, has its origin in the binding properties of the ionic core of the atom. This is demonstrated in Figures 5 and 6. 


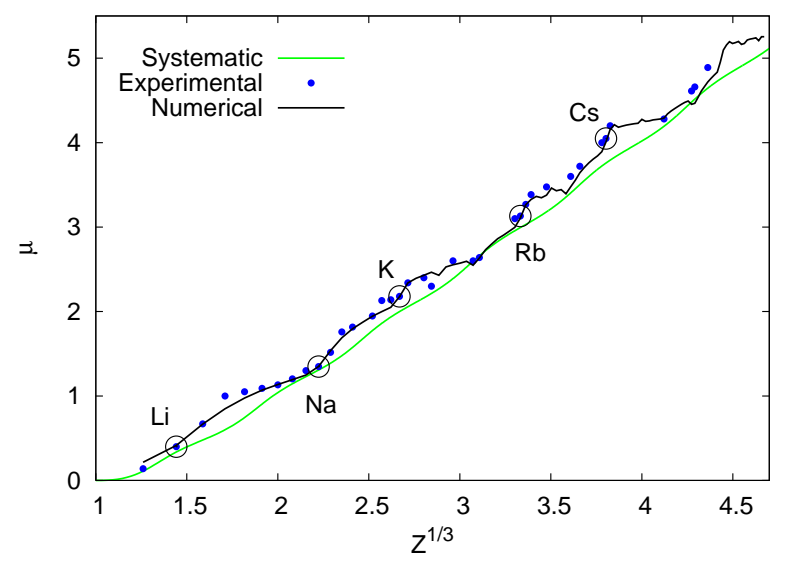

Figure 5: Systematic, experimental, and numerical dependences of the quantum defect $\mu$ on $Z^{1 / 3}$. To help orientation within the Periodic Table experimental alkali data are circled.

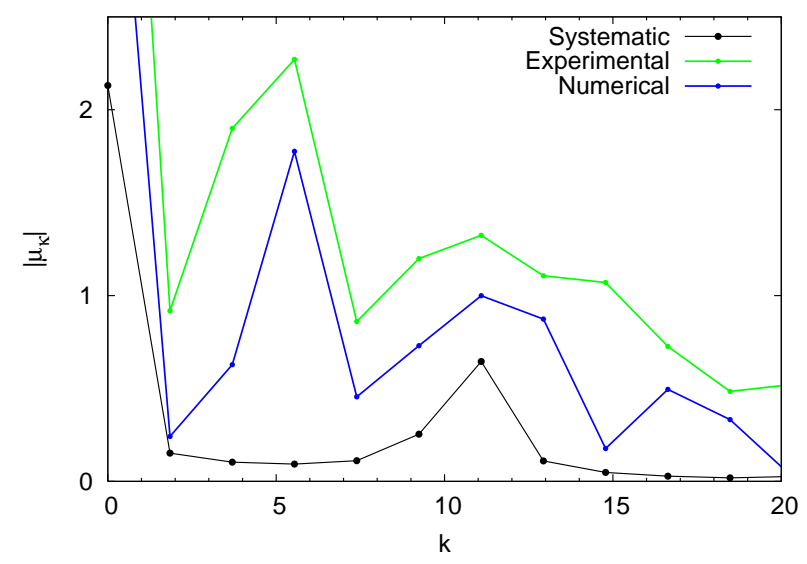

Figure 6: Systematic, experimental and numerical amplitudes of the Fourier coefficients of the quantum defect variation (arbitrary units, same normalization ) $\left|\mu_{k}\right|$ as functions of $k$ for $k \geq 0$. The peaks at $k \simeq 11$ correspond to the Zel'dovich effect. 


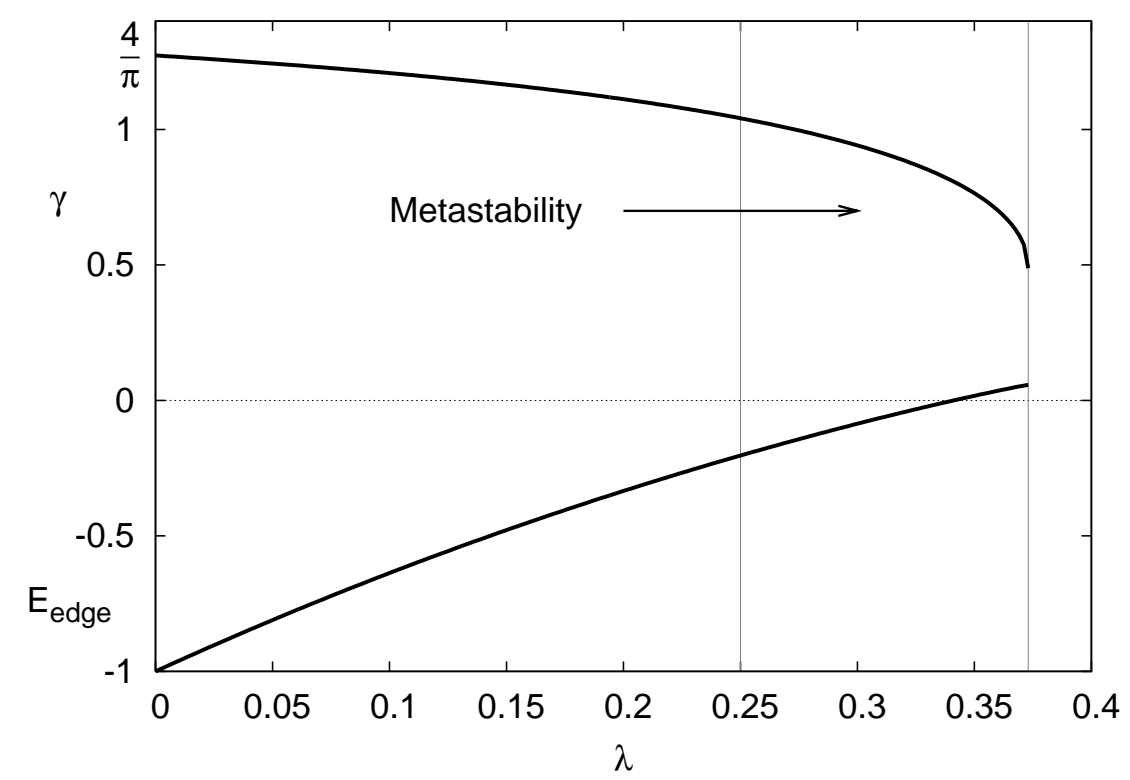

Figure 7: Dimensionless stiffness of the edge bond $\gamma$ and corresponding binding energy of the edge particle $E_{\text {edge }}$ of a half-infinite Luttinger liquid as functions of the quantum parameter $\lambda$. The region of metastability is confined to the $1 / 4 \leq \lambda \leq 0.3730$ range.

A more specific account of work submitted for publication supported by the current Department of Energy Research Grant is given below:

- Xiao Yang and Chetan Nayak, Ferromagnetism of Weakly-Interacting Electrons in Disordered Systems, submitted to Phys. Rev. B (2005), http://arxiv.org/abs/cond-mat/0501431. It was realized two decades ago that the two-dimensional diffusive Fermi liquid phase is unstable against arbitrarily weak electron-electron interactions. Recently, using the nonlinear sigma model developed by Finkelstein, several authors have shown that the instability leads to a ferromagnetic state. This paper considers diffusing electrons interacting through a ferromagnetic exchange interaction. Using the Hartree-Fock approximation to directly calculate the electron self-energy, it is found that the total energy is minimized by a finite ferromagnetic moment for arbitrarily weak interactions in two dimensions and for interaction strengths exceeding a critical value proportional to the conductivity in three dimensions. The relation between these and previous results is discussed.

- E. B. Kolomeisky and M. Timmins, Quantum dissociation of an edge of a Luttinger liquid, submitted to Phys. Rev. Lett. (2005), http://arxiv.org/abs/cond-mat/0506375. In a Luttinger liquid phase of one-dimensional molecular matter the strength of zero-point motion can be characterized by the dimensionless De Boer's number quantifying the interplay of quantum fluctuations and two-body interactions. Selecting the latter in the Morse form we show that dissociation of the Luttinger liquid is a process initiated at the system edge. The latter becomes unstable against quantum fluctuations at a value of De Boer's number which is smaller than that of the bulk instability which parallels the classical phenomenon of surface melting. These results are summarized in Figures 7, 8, and 9. 


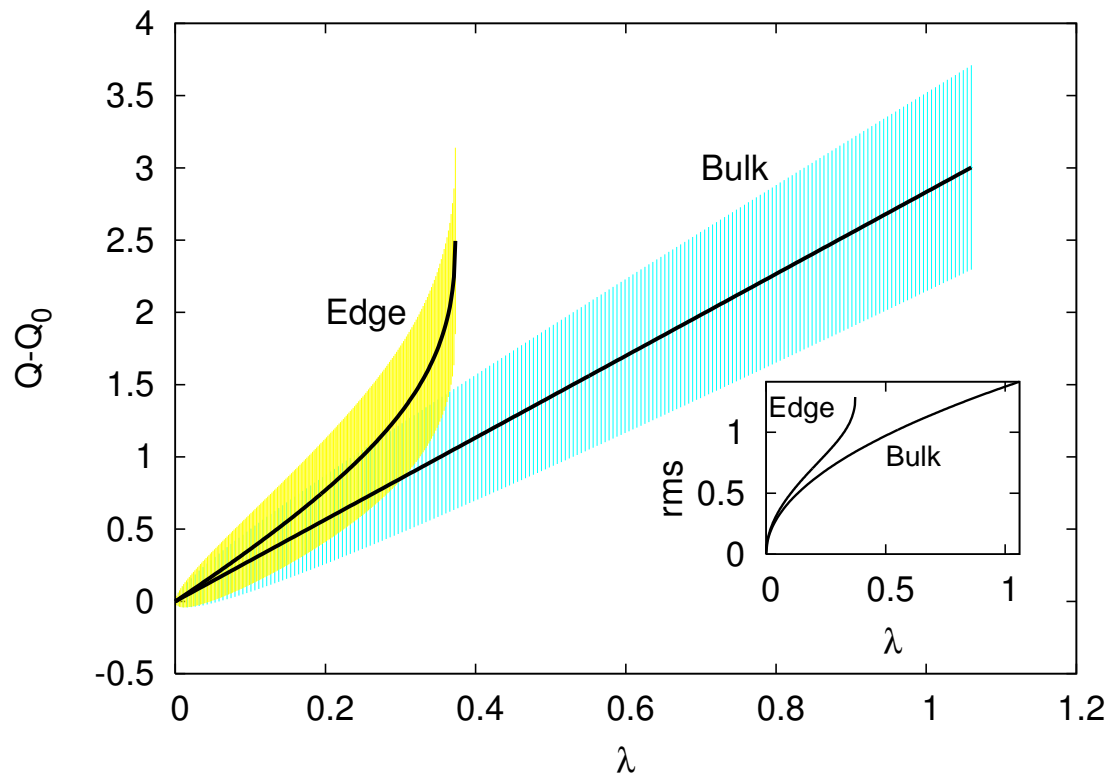

Figure 8: Dimensionless uantum expansion $Q-Q_{0}$ and rms fluctuation of the bulk and edge bonds as functions of the quantum parameter $\lambda$. The rms fluctuation is shown both as the vertical extent of shaded regions centered around the quantum expansion curves, and explicitly in the inset.

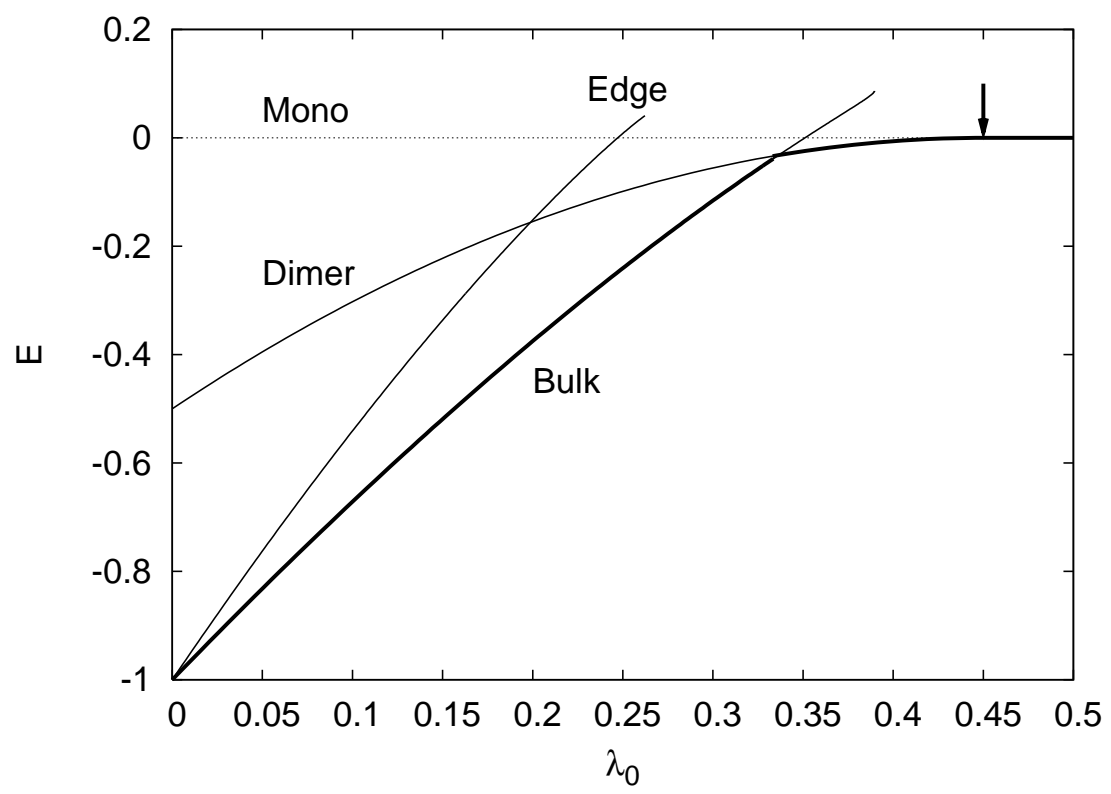

Figure 9: The dependences of the energy per particle (normalized to the classical case) for various bulk phases of the system on De Boer's number $\lambda_{0}$ together with edge binding energy. The arrow pointing down is the dimer dissociation threshold. 


\section{References}

[1] S. Iijima, Helical microtubules of graphitic carbon, Nature (London) 354, 56 (1991).

[2] P. M.Ajayan and T. W. Ebbesen, Nanometre-size tubes of carbon, Rep. Prog. Phys. 60, 1025 (1997).

[3] W. Teizer, R. B. Hallock, E. Dujardin, and T. W. Ebbesen, ${ }^{4} \mathrm{He}$ desorption from single wall carbon nanotube bundles: a one-dimensional adsorbate, Phys. Rev. Lett. 82, 5305 (1999), and references therein.

[4] A. C. Dillon, K. M. Jones, T. A. Bekkedahl, C. H. Kiang, D. S. Bethune, and M. J. Heben, Storage of hydrogen in single-walled carbon nanotubes, Nature (London) 386, 377 (1997); F. Darkrim and D. Levesque, Monte Carlo simulations of hydrogen adsorption in single-walled carbon nanotubes, J. Chem. Phys. 109, 4981 (1998); J. M. Ogden, Hydrogen: The fuel of the future?, Physics Today 55, 69 (2002), and references therein.

[5] J. De Boer, Quantum theory of condensed permanent gases I: the law of corresponding states, Physica 14, 139 (1948); J. De Boer and B. S. Blaisse, Quantum theory of condensed permanent gases II: the solid state and the melting line, Physica 14, 149(1948); J. de Boer and R. J. Lunbeck, Quantum theory of condensed permanent gases III: the equation of state of liquids, Physica 14, 520 (1948).

[6] P. W. Anderson and R. G. Palmer, Solidification pressure of nuclear and neutron star matter, Nat. Phys. Sci. 231, 145 (1971); Corresponding-states approach to nuclear and neutron-star matter, Phys. Rev. D 9, 3281 (1974); see also P. W. Anderson, Basic Notions of Condensed Matter Physics (Addison-Wesley Reading, Massachusetts, 1997), Section IIID.

[7] J. W Clark and N. C. Chao, The crystallization of neutronic matter, Nat. Phys. Sci. 236, 37 (1972).

[8] F. Lindemann, Über die Berechnung molekularer Eigenfrequenzen, Phys. Z. 11, 69 (1910).

[9] L. Khaykovich, F. Schreck, G. Ferrari, T. Bourdel, J. Cubizolles, L. D. Carr, Y. Castin, and C. Salomon, Formation of a matter-wave bright soliton, Science 296, 1290 (2002); K. E. Strecker, G. B. Partridge, A. G. Truscott, and R. G. Hulet, Formation and propagation of matter-wave soliton trains, Nature 417, 150 (2002). 